\title{
KDE-AFFECT at SemEval-2018 Task 1: Estimation of Affects in Tweet by Using Convolutional Neural Network for $n$-gram
}

\author{
Shinnosuke Himeno and Masaki Aono \\ Department of Computer Science and Engineering \\ Toyohashi University of Technology \\ himeno@kde.cs.tut.ac.jp, aono@tut.jp
}

\begin{abstract}
This paper describes our approach to SemEval-2018 Task1: Estimation of Affects in Tweet for $1 \mathrm{a}$ and $2 \mathrm{a}$. Our team KDE-AFFECT employs several methods including one-dimensional Convolutional Neural Network for $n$-grams, together with word embedding and other preprocessing such as vocabulary unification and Emoji conversions into four emotional words.
\end{abstract}

\section{Introduction}

With the rapid spread of SNS services (e.g. Twitter, Facebook, Instagram), massive user opinions have accumulated on the Internet. Among such opinions, it has been observed that not a few SNS contents naturally entail the affects (including joy, anger, sadness, fear) within themselves. Hence, the need to accurately detect the affects is increasing year by year.

In SemEval-2018 Task 1: Estimation of Affects in Tweet, we have attempted to extend our horizon from positive, neutral, and negative polarity estimations in former SemEval sentiment analysis in tweet having been held till 2017, to multiple emotions (joy, anger, sadness, and fear) in terms of regression (Task-1 1a) and classification (Task-1 2a). In doing so, we have adopted a standard one-dimensional Convolutional Neural Network $(\mathrm{CNN})$, which is believed to be effective for text polarity estimation, where the kernel window size for 1D convolution is analogous to the concept of word $n$-gram. In addition, as most people have noticed, a tweet has potentially many Emojis to express emotions. In the following, we first briefly survey related work on tweet sentiment analysis including emotion estimation. Then, we describe our system, followed by showing the results returned from the organizer, and finally concluding our paper.

\section{Related Work}

Sentiment analysis of tweets has been studied by many researchers from the standpoint of classifying a tweet into either positive or negative polarity, and classifying it into multiple emotions (Giachanou and Crestani, 2016; Silva et al., 2016). A supervised approach to polarity classification of a tweet was proposed by Go et al. (2009). They employed Naive Bayes, Maximum Entropy, Support Vector Machine, and several other machine learning methods for their supervised learning. Bravo-Marque et al. (2013) presented an approach using multiple emotion dictionaries, while Saif et al. (2016) employed cooccurrence information of words. Severyn et al. (2015) introduced a deep learning approach. Lu et al. (2013) proposed a deep learning method suited for short texts. In SemEval, since 2014, sentiment analysis tasks using Twitter have been officially conducted, where a variety of methods have been tested (Hagen et al., 2015; Giorgis et al., 2016; Deriu et al., 2016; Rouvier and Favre, 2016; $\mathrm{Xu}$ et al., 2016). In SemEval2017 Rosenthal et al. (2017), Cliche et al. (2017) and Hamdan et al. (2017) presented methods for combining multiple Convolutional Neural Networks (CNNs) and multiple Long Short-Term Memories (LSTMs). Mohammad (2017) published an open dictionary of emotion scores for each word. Mohammad et al. published a dataset for estimating emotion intensities (Mohammad and Bravo-Marquez, 2017).

\section{Methodology}

In this section, we focus on our methods and ideas employed in this task. The fundamental idea of our method is based on the observation that " $n$ grams" seem to have vital effects to represent the emotion of a tweet, where " $n$-gram" denotes $n$ consecutive words (instead of $n$ consecutive char- 


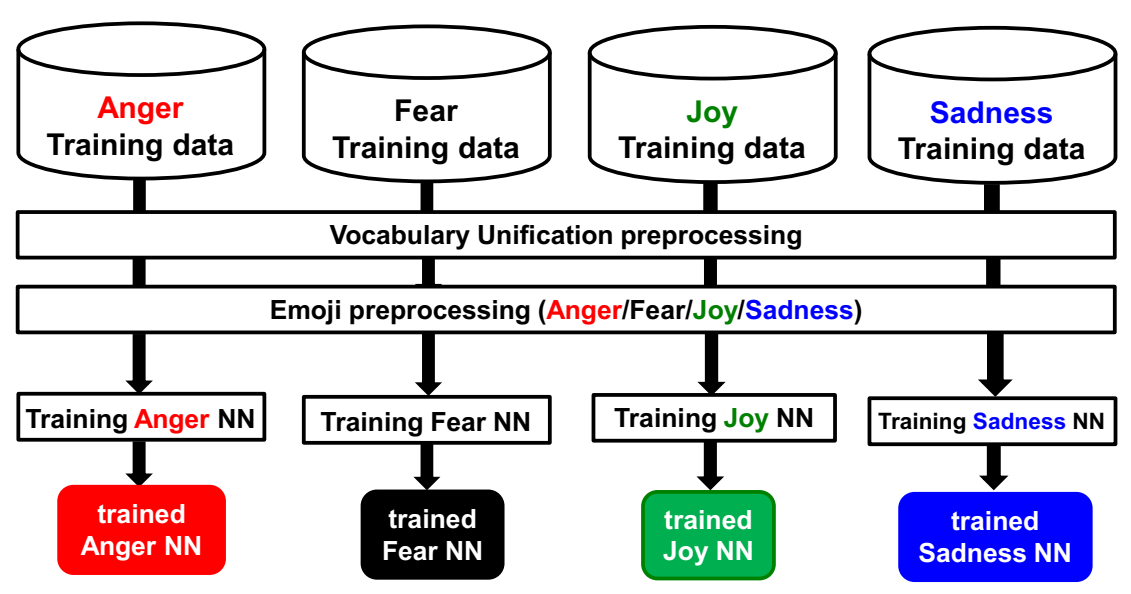

Figure 1: Overall Training Flow of KDE-AFFECT

acters). For instance, if the tweet sentence is "At last I made it.", 2-gram includes (At, last) and (made, it). Similarly, 3-gram includes (At, last, I) and (I, made, it).

We have adopted the method based on the $n$ gram convolution proposed by Kim (2014). Here, we prepare a matrix corresponding to a sentence representing an $n$-gram convolution in which this filtering process is carried out by the unit of an $n$ gram.

The overview of our system is as follows: First we apply preprocessing with "vocabulary unification" including lower case conversion, URL unification, two or more consecutive character squeezing, and hashtag elimination. Second, we apply Emoji conversion into four emotional words, which will be elaborated later. From Emoji conversion, we train the model independently for each emotion. Finally, we predict the emotion score for an unknown tweet by using the trained model. The overall system flow is shown in Figure 1.

\subsection{Preprocessing with Vocabulary Unification}

This step is applied to all emotions. It consists of the following processing:

- lower case conversion

- conversion of every instance of a URL string in a tweet to " $<$ URL $>$ "

- collapse of two or more consecutive letters into two

- elimination of hash sign (\#)

It should be noted that by a url string we mean a regular expression starting with either "http", "https", "ftp", or "www". Any url string is converted to $<\mathrm{URL}>$. For example, "I want to be happy on http://t.co/S6moxr1U" is converted to "I want to be happy on $<$ URL $>$ ".

\subsection{Preprocessing for Emoji}

From our observation of real tweets, approximately more than $20 \%$ of them have some kind of Emojis. Emotions are naturally represented by many different Emojis. Hence, we introduce the conversion of possible emotions represented by an Emoji into each emotional word. Please note that Emoji preprocessing is applied to all Emoji data, regardless of emotions. For instance, each anger Emoji might appear not only in an Anger dataset, but in Fear, Joy, and Sadness datasets as well. This is why we have decided to apply the Emoji conversion despite the differences of emotions. In the following, we present Emoji for each emotion, where Emoji has been taken from a Full Emoji Web site ${ }^{1}$. The selection of Emoji has been made by using the labels (such as "face-positive") annotated to the above Web sites.

\subsubsection{Anger Emoji}

The Anger Emojis we selected are shown in Figure 2. All of them are replaced by "anger".

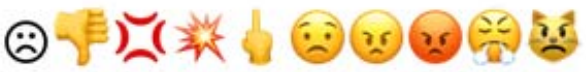

Figure 2: Anger Emoji

\subsubsection{Fear Emoji}

The Fear Emojis we selected are shown in Fig. 3. All of them are replaced by "fear".

\footnotetext{
${ }^{1}$ https://unicode.org/emoji/charts/full-emoji-list.html
} 


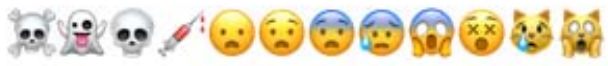

Figure 3: Fear Emoji

\subsubsection{Joy Emoji}

The Joy Emojis we selected are shown in Fig. 4. All of them are replaced by "joy".

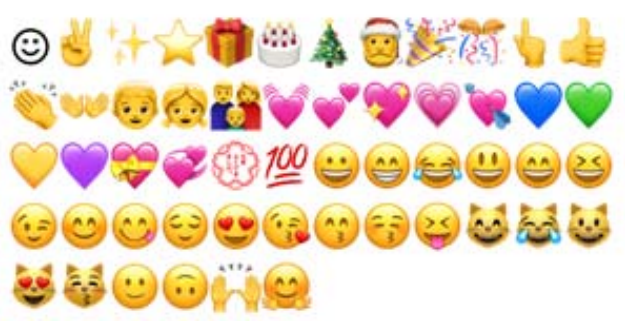

Figure 4: Joy Emoji

\subsubsection{Sadness Emoji}

The Sadness Emojis we selected are shown in Fig. 5. All of them are replaced by "sadness".

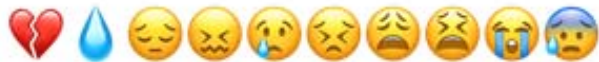

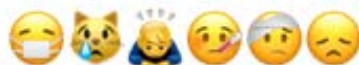

Figure 5: Sadness Emoji

\subsection{Convolutional Neural Network for $n$-gram}

Once preprocessing is done, we have a kind of rectified tweet, represented by a matrix. Figure 6 illustrates a word-by-word matrix representation of a rectified tweet. Here we take a matrix of 80 by 300 , where 80 is the maximum number of words per tweet, and 300 corresponds to our embedding vector size. If a tweet has less than 80 words, zero padding is performed to fill the input matrix.

\subsubsection{Embedding}

In Embedding, each tweet is converted to a matrix. Specifically, we first divide a tweet into words using a whitespace, thereby treating a special character (one of ".h, ",h, "!h, and "?h ) as a separate word. Second, we transform each word into its distributed representation of 300 dimensions using Word2Vec (Mikolov et al., 2013a,b). The training of Word2Vec itself is done by using approximately 470 million tweets after the processing de-

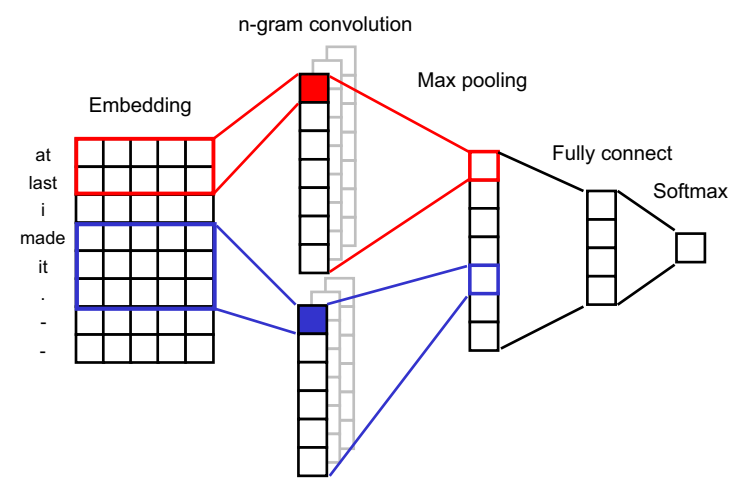

Figure 6: Our $n$-gram Convolution-based Approach

scribed in 3.1. We finally obtain the embedding by padding zero values to a fixed size of a 80 by 300 dimensional matrix.

\subsection{2 $n$-gram Convolution Layer}

In an $n$-gram convolutional layer, we perform convolution, and generate a length $m-n+1$ vector, where $m$ denotes the maximum word length (here 80). This is straightforward, since both ends are trimmed during the $n$-gram convolution stage shown in Figure 6. For instance, if 3-gram is concerned, the length in our implementation will be $80-3+1=78$. Note that we have multiple $n$-gram convolutional layers for each emotion. "Joy" neural network architecture, for example, has 2-gram, 3-gram, 4-gram, and 5-gram convolutional layers, which will be discussed later in Table 3 .

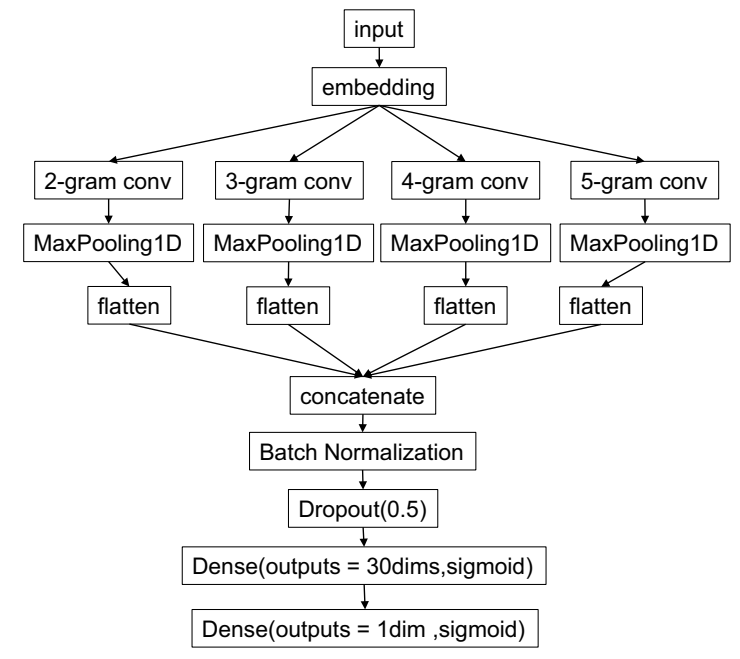

Figure 7: KDE-AFFECT system's DNN architecture

\subsubsection{Max Pooling Layer}

In a Max Pooling layer, from each $n$-gram convolutional layer, the maximum value is computed, 


\begin{tabular}{|l|l|}
\hline Intensity range & Intensity amount \\
\hline$[0.0,0.35)$ & 0 (no E) \\
{$[0.35,0.5)$} & 1 (low amount of $\mathrm{E})$ \\
{$[0.5,0.65)$} & 2 (moderate amount of $\mathrm{E})$ \\
{$[0.65,1.0]$} & 3 (high amount of $\mathrm{E})$ \\
\hline
\end{tabular}

Table 1: Inferred Intensity Level

\begin{tabular}{|c|c|c|c|}
\hline & Anger Fear & Joy & Sadness \\
\hline Training & $1701 \quad 2252$ & 1616 & 1532 \\
\hline Dev. & $\overline{388}, \overline{389}-$ & $\overline{2} 9 \overline{0}$ & $-\overline{39} \overline{7}$ \\
\hline$\overline{\text { Test }}(\overline{1} \bar{a})$ & $-1 \overline{7} \overline{940}, \overline{17} \overline{92} \overline{4}$ & $\overline{1} \overline{8} 0 \overline{4} \overline{3}$ & $-\overline{17} \overline{13} \overline{3}-$ \\
\hline$\overline{\text { Test }}(\overline{2} \bar{a})$ & $1002 \quad, \quad \overline{9} 86$ & $1 \overline{1} 05^{-}$ & $\overline{9} \overline{7} \overline{5}^{-}$ \\
\hline
\end{tabular}

Table 2: SemEval 2018: Task 1 dataset (1a and 2a)

\subsection{Evaluation Measure}

Here, the evaluation measure for a model is correlation coefficient $r$. Given variables $x$ and $y$, where $x$ corresponds to a predicted emotion value and $y$ to a true emotion value, and their associated sample variances $S_{x}, S_{y}$, and the covariance $S_{x y}$ are represented by the following equation:

$$
r=\frac{S_{x y}}{S_{x} S_{y}}
$$

\subsection{Experiment Environment}

For our deep learning program for the task, we used the following list of hyper-parameters:

loss function: Root Mean Square Error (RMSE)

filter number: $200 \times n$

epochs: 30

dropout rate: 0.5

optimizer: Adam

batch size: 64

The framework we use is Keras with backend Tensorflow. In our Ubuntu server, it took approximately 1 second for each epoch.

\subsection{Preliminary Experiments for $n$-gram Convolutions}

For each emotion, our system attempts to find an empirical optimal combination of $n$-gram convolutions. Table 3 summarizes the results of preliminary experiments for this purpose. Here, $r(A)$ denotes the correlation coefficient for Anger. Similarly, $r(F)$ for Fear, $r(J)$ for Joy, and $r(S)$ for Sadness. From the table, we decided as follows: For Anger, we chose $[1,2,3,4,5,6]$ (meaning we took the combination of 1-gram, 2-gram, 3-gram, 4-gram, 5-gram, and 6-gram convolutions). For Fear, we chose $[2,3,4,5,6]$. For Joy and Sadness, we chose $[2,3,4,5]$.

\subsection{Experimental Result (Task 1a)}

According to the Official Leaderboard for Task 1a, our team KDE-AFFECT turned out to be 30-th. If 


\begin{tabular}{c|cccc}
\hline$n$ & $r(A)$ & $r(F)$ & $r(J)$ & $r(S)$ \\
\hline$[1,2,3,4,5,6]$ & $\mathbf{0 . 5 5 2 9}$ & 0.5919 & 0.5771 & 0.6349 \\
{$[2,3,4,5,6]$} & 0.5518 & $\mathbf{0 . 5 9 9 4}$ & 0.5464 & 0.6173 \\
{$[1,2,3,4,5]$} & 0.5381 & 0.5948 & 0.5730 & 0.6078 \\
{$[2,3,4,5]$} & 0.5309 & 0.5736 & $\mathbf{0 . 5 9 0 6}$ & $\mathbf{0 . 6 3 6 0}$ \\
\hline
\end{tabular}

Table 3: Preliminary experiments for $n$-gram convolustions

\begin{tabular}{c|ccccc}
\hline Team & avg- $r$ & $r(A)$ & $r(F)$ & $r(J)$ & $r(S)$ \\
\hline KDE-AFFECT & 0.620 & 0.630 & 0.621 & 0.598 & 0.630 \\
SeerNet $^{1 s t}$ & 0.799 & 0.827 & 0.799 & 0.792 & 0.798 \\
NTUA-SLP $^{2 n d}$ & 0.776 & 0.782 & 0.758 & 0.771 & 0.792 \\
PlusEmo2Vec $^{3 r d}$ & 0.766 & 0.811 & 0.728 & 0.773 & 0.753 \\
CrystalFeel $^{14 t h}$ & 0.717 & 0.740 & 0.700 & 0.708 & 0.720 \\
EliRF-UPV $^{15 t h}$ & 0.696 & 0.705 & 0.686 & 0.693 & 0.700 \\
iit_delhi $^{29 t h}$ & 0.621 & 0.633 & 0.645 & 0.618 & 0.588 \\
DeepMiner $^{31 t h}$ & 0.575 & 0.581 & 0.570 & 0.575 & 0.573 \\
Baseline $^{37 t h}$ & 0.520 & 0.526 & 0.525 & 0.575 & 0.453 \\
\hline
\end{tabular}

Table 4: Our result with selected other teams for Task 1a

\begin{tabular}{c|ccccc}
\hline Team & avg- $r$ & $r(A)$ & $r(F)$ & $r(J)$ & $r(S)$ \\
\hline KDE-AFFECT $^{\prime}$ & 0.530 & 0.530 & 0.470 & 0.552 & 0.567 \\
SeerNet $^{1 s t}$ & 0.695 & 0.706 & 0.637 & 0.720 & 0.717 \\
PlusEmo2Vec $^{2 n d}$ & 0.659 & 0.704 & 0.528 & 0.720 & 0.683 \\
psyML $^{3 r d}$ & 0.653 & 0.670 & 0.588 & 0.686 & 0.667 \\
UNCC $^{9 t h}$ & 0.599 & 0.604 & 0.544 & 0.638 & 0.610 \\
ECNU $^{16 t h}$ & 0.531 & 0.565 & 0.441 & 0.581 & 0.536 \\
CrystalFeel $^{18 t h}$ & 0.530 & 0.576 & 0.466 & 0.540 & 0.538 \\
Baseline $^{26 t h}$ & 0.394 & 0.382 & 0.355 & 0.496 & 0.370 \\
\hline
\end{tabular}

Table 5: Our result with selected other teams for Task 2a

we use similar notations as in Table 3, and pick up the top- 3 ranked teams, as well as randomly chosen teams CrystalFeel (14-th place), ELipRF-UPV (15-th place), iit_delhi (29-th), DeepMiner (31-th), and the baseline (37-th), the result looks like Table 4 .

\subsection{Experimental Result (Task 2a)}

According to the Official Leaderboard for Task 2a, our team KDE-AFFECT turned out to be 17-th. If we use similar notations as in Table 3, and pick up top-3 ranked teams, as well as randomly chosen teams UNCC (9-th place), ECNU (16-th place), CrystalFeel (14-th place), and the baseline (26-th), the result looks like Table 5.

\section{Conclusion}

This paper describes the approach we took for SemEval-2018 Task 1: Affect in Tweets (subtasks 1a and 2a). We have chosen a combination of different $n$-gram convolutions with preprocessing including vocabulary unification and Emoji conversion.

\section{Acknowledgments}

Part of this research is supported by MEXT KAKENHI, Grant-in-Aid for Scientific Research (B), Grant Number 17 H01746.

\section{References}

Felipe Bravo-Marquez, Marcelo Mendoza, and Barbara Poblete. 2013. Combining strengths, emotions and polarities for boosting twitter sentiment analysis. In Proceedings of the Second International Workshop on Issues of Sentiment Discovery and Opinion Mining, WISDOM '13, pages 2:1-2:9, Chicago, Illinois. 
Mathieu Cliche. 2017. Bb_twtr at semeval-2017 task 4: Twitter sentiment analysis with cnns and lstms. In Proceedings of the 11th International Workshop on Semantic Evaluation (SemEval-2017), pages 573580, Vancouver, Canada. Association for Computational Linguistics.

Jan Deriu, Maurice Gonzenbach, Fatih Uzdilli, Aurelien Lucchi, Valeria De Luca, and Martin Jaggi. 2016. Swisscheese at semeval-2016 task 4: Sentiment classification using an ensemble of convolutional neural networks with distant supervision. In Proceedings of the 10th International Workshop on Semantic Evaluation (SemEval-2016), pages 11241128, San Diego, California. Association for Computational Linguistics.

Anastasia Giachanou and Fabio Crestani. 2016. Like it or not: A survey of twitter sentiment analysis methods. ACM Computing Surveys, 49(2):28:1-28:41.

Stavros Giorgis, Apostolos Rousas, John Pavlopoulos, Prodromos Malakasiotis, and Ion Androutsopoulos. 2016. aueb.twitter.sentiment at semeval-2016 task 4: A weighted ensemble of svms for twitter sentiment analysis. In Proceedings of the 10th International Workshop on Semantic Evaluation (SemEval2016), pages 96-99, San Diego, California. Association for Computational Linguistics.

Alec Go, Richa Bhayani, and Lei Huang. 2009. Twitter sentiment classification using distant supervision. Processing, pages 1-6.

Matthias Hagen, Martin Potthast, Michel Büchner, and Benno Stein. 2015. Webis: An ensemble for twitter sentiment detection. In Proceedings of the 9th International Workshop on Semantic Evaluation (SemEval 2015), pages 582-589, Denver, Colorado. Association for Computational Linguistics.

Hussam Hamdan. 2017. Senti17 at semeval-2017 task 4: Ten convolutional neural network voters for tweet polarity classification. In Proceedings of the 11th International Workshop on Semantic Evaluation (SemEval-2017), pages 700-703, Vancouver, Canada. Association for Computational Linguistics.

Yoon Kim. 2014. Convolutional neural networks for sentence classification. In Proceedings of the 2014 Conference on Empirical Methods in Natural Language Processing, EMNLP 2014, October 25-29, 2014, Doha, Qatar, A meeting of SIGDAT, a Special Interest Group of the ACL, pages 1746-1751.

Zhengdong Lu and Hang Li. 2013. A deep architecture for matching short texts. In Advances in Neural Information Processing Systems 26, pages 1367-1375. Curran Associates, Inc.

Tomas Mikolov, Kai Chen, Greg Corrado, and Jeffrey Dean. 2013a. Efficient estimation of word representations in vector space. CoRR, abs/1301.3781.
Tomas Mikolov, Ilya Sutskever, Kai Chen, Greg S Corrado, and Jeff Dean. 2013b. Distributed representations of words and phrases and their compositionality. In C. J. C. Burges, L. Bottou, M. Welling, Z. Ghahramani, and K. Q. Weinberger, editors, $A d$ vances in Neural Information Processing Systems 26, pages 3111-3119. Curran Associates, Inc.

Saif Mohammad and Felipe Bravo-Marquez. 2017. Emotion intensities in tweets. In Proceedings of the 6th Joint Conference on Lexical and Computational Semantics (*SEM 2017), pages 65-77. Association for Computational Linguistics.

Saif M. Mohammad. 2017. Word affect intensities. CoRR, abs/1704.08798.

Saif M. Mohammad, Felipe Bravo-Marquez, Mohammad Salameh, and Svetlana Kiritchenko. 2018. Semeval-2018 Task 1: Affect in tweets. In Proceedings of International Workshop on Semantic Evaluation (SemEval-2018), New Orleans, LA, USA.

Sara Rosenthal, Noura Farra, and Preslav Nakov. 2017. Semeval-2017 task 4: Sentiment analysis in twitter. In Proceedings of the 11th International Workshop on Semantic Evaluation (SemEval-2017), pages 502-518. Association for Computational Linguistics.

Mickael Rouvier and Benoit Favre. 2016. Sensei-lif at semeval-2016 task 4: Polarity embedding fusion for robust sentiment analysis. In Proceedings of the 10th International Workshop on Semantic Evaluation (SemEval-2016), pages 202-208, San Diego, California. Association for Computational Linguistics.

Hassan Saif, Yulan He, Miriam Fernandez, and Harith Alani. 2016. Contextual semantics for sentiment analysis of twitter. Information Processing and Management, 52(1):5-19.

Aliaksei Severyn and Alessandro Moschitti. 2015. Twitter sentiment analysis with deep convolutional neural networks. In Proceedings of the 38th International ACM SIGIR Conference on Research and Development in Information Retrieval, SIGIR '15, pages 959-962, Santiago, Chile. ACM.

Nadia Felix F. Da Silva, Luiz F. S. Coletta, and Eduardo R. Hruschka. 2016. A survey and comparative study of tweet sentiment analysis via semi-supervised learning. ACM Comput. Surv., 49(1):15:1-15:26.

Steven Xu, HuiZhi Liang, and Timothy Baldwin. 2016. Unimelb at semeval-2016 tasks 4a and 4b: An ensemble of neural networks and a word2 $\mathrm{vec}$ based model for sentiment classification. In Proceedings of the 10th International Workshop on Semantic Evaluation (SemEval-2016), pages 183-189, San Diego, California. Association for Computational Linguistics. 